\title{
Is the presence of echo-rich periportal cuffing in the liver indicator for abdominal inflammation in pediatric patients?
}

\author{
Nurdan Fidan ${ }^{1}$, Esra Ummuhan Mermi Yetis ${ }^{2}$, Muammer Murat ${ }^{1}$, Cuneyt Yucesoy ${ }^{3}$, Ebru \\ Turgal $^{4}$, Mehmet Metin 5
}

${ }^{1}$ Department of Radiology, Hitit University Erol Olcok Training and Research Hospital, Corum, ${ }^{2}$ Department of Radiology, Maltepe University Faculty of Medicine, Istanbul, ${ }^{3}$ Department of Radiology, Hitit University Faculty of Medicine, Corum, ${ }^{4}$ Department of Biostatistics, Hitit University Faculty of Medicine, Corum, ${ }^{5}$ Department of Pediatric Surgery, Hitit University Erol Olcok Training and Research Hospital, Corum, Turkey

\begin{abstract}
Aims: Hyperechoic/echo-rich periportal cuffing (ErPC) is defined as an increase in echogenicity relative to the adjacent liver parenchyma. Thickening in the periportal area may occur with proliferation of bile ducts, hemorrhage, oedema, fibrosis, inflammatory changes or a combination of these. The aim of this study is to determine which intraabdominal inflammatory diseases are associated with the presence of ErPC in the pediatric population and to calculate the sensitivity and specificity of this finding. Material and methods: In this prospective study 200 consecutive children who underwent abdominal ultrasonography (US) were included: group 1, the patient group (100 children with appendicitis, gastroenteritis, mesenteric lymphadenitis, intestinal infection, terminal ileitis and invagination as cause of intra-abdominal inflammation) and group 2, the control group (100 children). Results: The ErPC was positive in 74 (74\%) cases in the patient group and in 3 (3\%) in the control group. According to final diagnoses, we found ErPC in most of patients with gastroenteritis (16/17), perforated appendicitis (10/11), mesenteric lymphadenitis (5/6) and acute appendicitis (27/37). The sensitivity of ErPC in indicating intra-abdominal inflammation was 0.80 and its specificity was 0.87 . No significant correlation between ErPC and age, gender and CRP was found but a moderate and significant positive correlation between ErPC and WBC $(p=0,010 ; r=0.255)$ was detected. Very good concordance between observers in terms of the presence of ErPC on abdominal US was found (concordance 97\% and kappa 0.93 ). Conclusions: We consider that the presence of ErPC in pediatric patients, when evaluated alongside clinical and laboratory findings, has a high sensitivity and specificity for inflammatory intra-abdominal pathology.

Keywords: periportal cuffing; inflammation; acute abdomen; ultrasound; children
\end{abstract}

\section{Introduction}

The periportal area is the anatomical area around the portal vein in the liver, including branches of the hepat-

Received 04.03.2019 Accepted 02.07.2019

Med Ultrason

2019, Vol. 21, No 3, 225-231

Corresponding author: Nurdan Fidan, MD

Department of Radiology, Hitit University

Erol Olcok Training and Research Hospital, 19200, Corum, Turkey

E-mail: kocak_nurdan@yahoo.com

Phone: +90 3642193000

Fax:0 3642233030 ic artery, bile duct, nerves, lymph ducts and potentially empty areas. Periportal pathology occurs when any of these structures are affected. The periportal area may become wider due to inflammatory processes, tumor infiltration, bile duct proliferation, hemorrhage or oedema and this appearance is radiologically defined as a periportal halo. Periportal halo is also referred as periportal cuffing (PC) in ultrasonography (US) and may appear as an increase or decrease of echogenicity in the periportal area. Hyperechoic/ echo-rich periportal cuffing (ErPC) is defined as an increase in echogenicity relative to the adjacent liver parenchyma and is termed "starry sky" or "stars and stripes" [1-5]. It generally appears with acute 
hepatitis, cholecystitis, pancreatitis, primary sclerosing cholangitis, pericolangitis, hepatic trauma, primary biliary cirrhosis and inflammatory bowel disease [6-11].

In previous studies, it has been mentioned that PC is a very rare ultrasonographic finding $(0.95 \%)[10,11]$. The pathogenesis of ErPC is not completely known and histological findings have not been reported [10]. Thickening in the periportal area may occur due to proliferation of the bile ducts, hemorrhage, oedema, fibrosis, inflammatory changes, or a combination of these $[9,12]$. It has been hypothesized that abnormal cells in inflammatory bowel disease emerge due to the transition of enterohepatic circulation from the intestinal mucosa into the portal system and inflammation of the liver [11].

We planned this study to answer the question of whether this finding may be related or not to intra-abdominal inflammation, because in our radiology unit we frequently observed periportal echogenicity in pediatric patients presenting with acute abdominal findings. In adult patients the association between periportal echogenicity and inflammatory diseases, such as inflammatory bowel diseases, hepatitis, pancreatitis, biliary tract diseases, parasitic disease or pyelonephritis, has been already described $[10,11]$. In the pediatric population, the association has been reported in patients with cystic fibrosis, hemophagocytic lymphohistiocytosis and appendicitis [12-18] but no primary study regarding specific inflammatory diseases related to ErPC in pediatric patients was published. The aim of this study is to determine which intra-abdominal inflammatory diseases are associated with the presence of ErPC in the pediatric population and to calculate the sensitivity and specificity of this finding for acute intra-abdominal pathologies.

\section{Materials and methods}

\section{Study population}

The study was conducted prospectively with the approval of the local Ethics Committee at the Radiology Department of Corum Training and Research Hospital, between 2015-2017. The study included 200 consecutive pediatric patients aged between 2 and 18 years old who were divided into two groups. In Group 1 (patient group) 100 patients referred by the emergency department for an abdominal US with pre-diagnosis of acute abdominal pain, acute abdomen, acute appendicitis, nausea, vomiting, diarrhea and invagination were enrolled. All these patients were hospitalized, followed up and treated. White blood cell count (WBC) in all patients and C-reactive protein (CRP) in 71 patients were measured. Values of $>10 \times 10^{9} / \mathrm{L}$ for $\mathrm{WBC}$ and $>5 \mathrm{mg} / \mathrm{L}$ for CRP were considered to be high. Group 2 (control group) consisted of
100 pediatric patients presented for abdominal US with pre-diagnosis of chronic abdominal pain (more than 3 weeks), urinary tract infection, hepatic steatosis/obesity, hematuria, dyspepsia, urolithiasis, enuresis, chronic epigastric pain, hepatosplenomegaly and hydronephrosis. WBC was determinated in all patients. No hospitalization was needed in this group.

The patients referred with a pre-diagnosis known to modify the periportal area such as trauma, viral hepatitis, pancreatitis, pyelonephritis, gallbladder/biliary tract pathology were excluded. In addition, we excluded patients with FMF (familial mediterranean fever) attack, ovarian torsion or ovarian cyst rupture, alterated liver tests or elevated serum amylase.

The demographic data (age, gender) and pre-diagnosis were collected. The patients were followed up until the final diagnosis and treatment was established.

\section{US evaluation}

US examinations were performed by a radiologist with 5 years of experience in pediatric US using Philips HD15 and Philips Affiniti 70G (Philips Healthcare, Amsterdam, the Netherlands) with a 3-5 MHz / 3-12 MHz frequency transducer. A second radiologist was consulted regarding the presence of ErPC in the liver without knowing the patient's pre-diagnosis and US results. The periportal echogenicity was compared with the posterior diaphragm echogenicity. ErPC was considered positive when there was an increase in echogenicity in the periportal area up to the diaphragm echo, diffuse (central and peripheric) and in both lobes [12] (fig 1-5).

In the US examination, if the anterior-posterior diameter of the appendix with a blind-ending, tubular and nonperistaltic segment of bowel that arises from the cecum was less than $6 \mathrm{~mm}$, it was interpreted as normal. If the appendix diameter was greater than $6 \mathrm{~mm}$ and non-

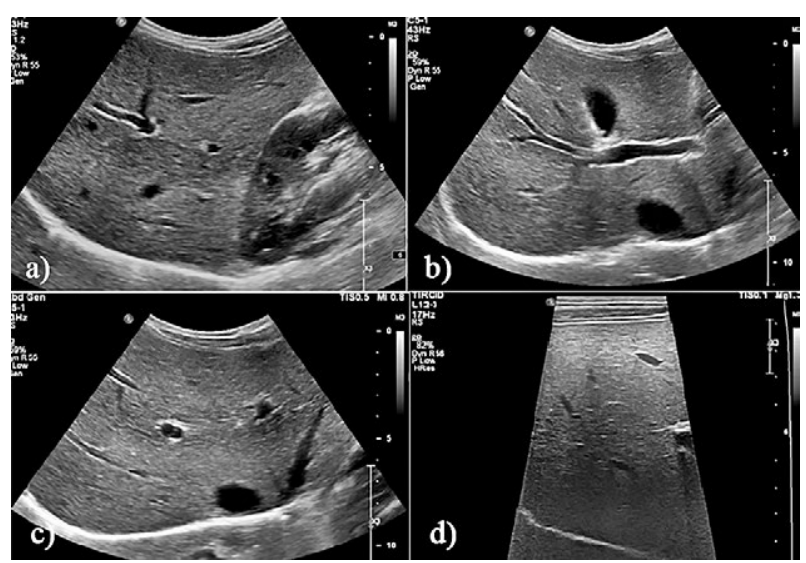

Fig 1. A 9-year-old boy with upper respiratory tract infection: a-c) US images with convex transducer of the normal liver; d) US image with high frequency linear-array transducer. 
compressible, it was interpreted as acute appendicitis $[19,20]$. The wall thickness of the bowels greater than 2 $\mathrm{mm}$ was interpreted as edema and dilatation of the bowel loops more than $2.5 \mathrm{~cm}$ in diameter was considered pathologic [21-23]. The presence of free fluid between the bowel loops and mesenteric lymph nodes (the size of the largest lymph node was measured) in the right lower quadrant of the abdomen were recorded. If the number of lymph nodes were $\geq 5$, with dimensions over $10 / 5 \mathrm{~mm}$ or clusters, the findings were considered to be positive in terms of mesenteric lymphadenitis [20,24,25].

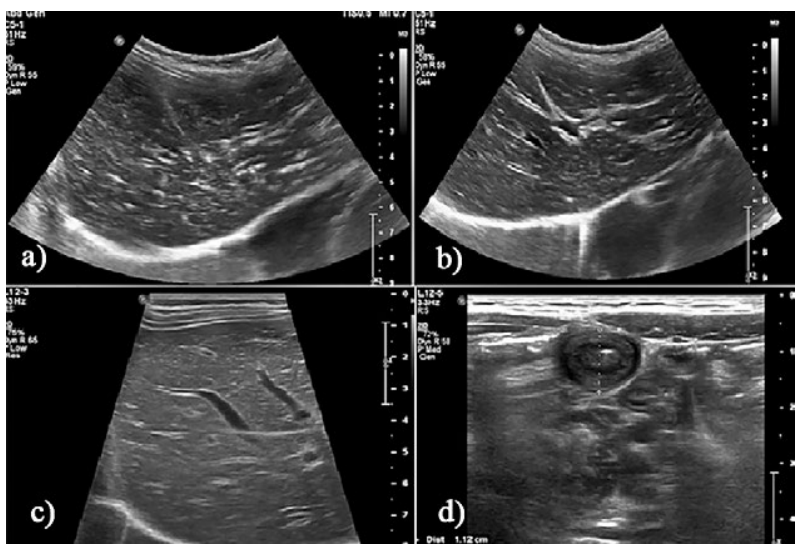

Fig 2. A 7-year-old girl with a history of acute abdominal pain: a-c) US images with convex transducer of the liver demonstrate increased periportal echogenicity in the central and peripheral parenchyma; d) US image with linear-array transducer of the right lower quadrant shows distended, noncompressible, abnormal appendix with thickened appendiceal wall due to inflammation. Final diagnosis was acute appendicitis.

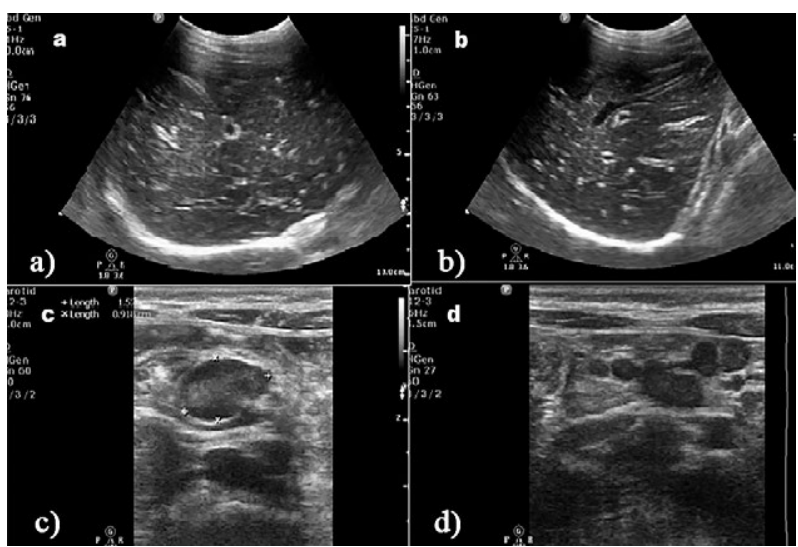

Fig 4. A 10-year-old boy with a history of acute abdominal pain: $a, b)$ US images of the liver demonstrate increased periportal echogenicity; c, d) US images with linear-array transducer of the right lower quadrant shows clustered, enlarged and tender mesenteric lymph nodes in the right lower quadrant with a long-axis diameter of $1.5 \mathrm{~cm}$. The final diagnosis was mesenteric lymphadenitis.

\section{Statistical analysis}

The data were summarized with descriptive statistics. Continuous quantitative variables are expressed in terms of mean and standard deviation values, and qualitative variables are expressed in terms of the minimum and maximum values. Categorical data were compared with chi-square and Fisher's exact tests. A point-biserial correlation coefficient was used to determine the relationship between binary and continuous variables. Phi coefficient was used for the association of two binary variables. Sensitivity and specificity for PC were calcu-

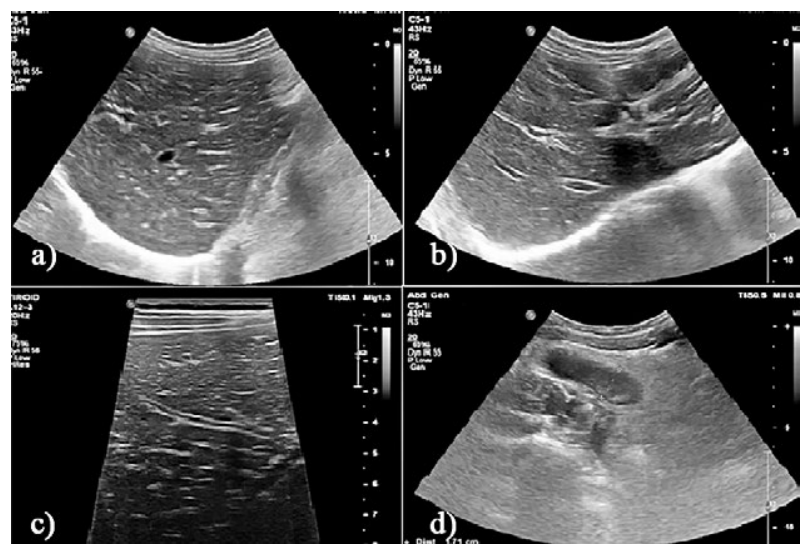

Fig 3. An 8-year-old girl with a history of acute abdominal pain and nausea: $a, b)$ US images with convex transducer of the liver demonstrate increased periportal echogenicity in the central and peripheral parenchyma; c) US image with high frequency linear-array transducer of the liver shows "starry sky appearance" of increased periportal echogenicity; d)US image of the right lower quadrant shows an abnormal appendix with adjacent increased echogenicity. Final diagnosis was perforated appendicitis.

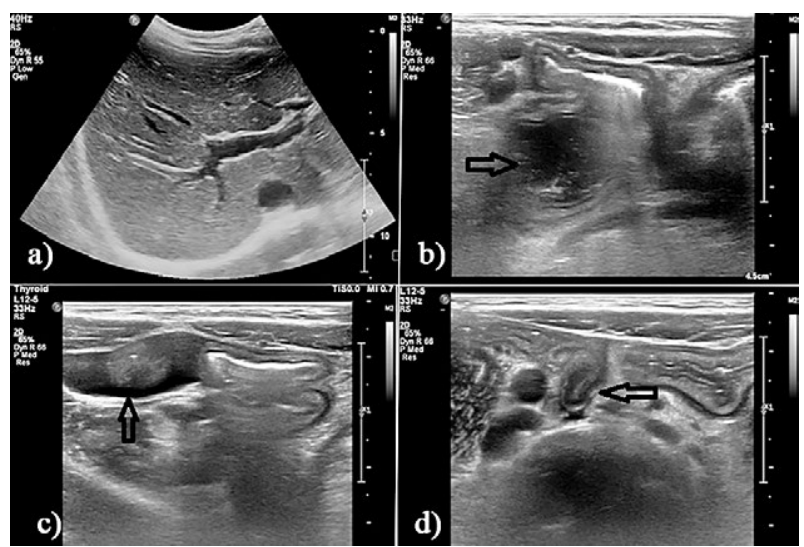

Fig 5. A 6-year-old boy with a history of acute abdominal pain, nausea and diarrhea: a) US image of the liver demonstrate increased periportal echogenicity; b-d) US images with linear-array transducer of the right lower quadrant shows the distended bowel loop(arrow), presence of free intraabdominal fluid (arrow) and the appendix is normal size (arrow). Final diagnosis was gastroenteritis. 
Table I. Summary of the demographic data and pre-diagnosis of the referred patients for abdominal ultrasonography

\begin{tabular}{llll}
\hline & Patient group (n:100) & Control group (n:100) & p value \\
\hline Age $($ years $)$ & $9.72 \pm 4.30$ & $8.5 \pm 3.79$ & $\mathrm{p}=0.066$ \\
Sex $(\mathrm{M} / \mathrm{F})$ & $67 / 33$ & $35 / 65$ & $<0.001$ \\
WBC $\left(\mathrm{x} 10^{9} / \mathrm{L}\right)$ & $14.87 \pm 6.13$ & $7.74 \pm 1.89$ & $<0.001$ \\
CRP $(\mathrm{mg} / \mathrm{L})$ & $41.62 \pm 62.97$ & - & Chronic abdominal pain -42 \\
Pre-diagnosis - number of cases & Acute appendicitis -49 & Urinary tract infection -24 \\
& Acute abdomen -28 & Hematuria -7 & Dyspepsia -7 \\
& Acute abdominal pain -15 & Urolithiasis -6 & \\
& Invagination -3 & Other* -11 & \\
\hline
\end{tabular}

WBC, white blood cell; CRP, C-reactive protein; *Other pre-diagnosis: enuresis nocturna, chronic epigastric pain, hepatosplenomegaly, hydronephrosis

Table II. The number (n) and percentage (\%) of ultrasongraphic findings in patient group

\begin{tabular}{ll}
\hline US findings & n (\%) \\
\hline Acute appendicitis & $47(47)$ \\
Mesenteric lymph nodes & $12(12)$ \\
Invagination & $3(3)$ \\
Perforated appendicitis & $3(3)$ \\
Thickening of the terminal ileum + & $3(3)$ \\
Mesenteric lymph nodes & \\
Acute appendicitis + & $2(2)$ \\
Mesenteric lymph nodes & \\
Perforated appendicitis + & $1(1)$ \\
Mesenteric lymph nodes & \\
Dilated bowel & $1(1)$ \\
Fluid in the right lower quadrant & $19(19)$ \\
Normal & $27(27)$ \\
Increased periportal echogenicity & $74(74)$ \\
\hline
\end{tabular}

lated. Kappa (K) was interpreted as an indication of poor agreement when less than 0.2 and as fair agreement when 0.20 . All tests were conducted at the two-sided $5 \%$ significance level using SPSS 20.

\section{Results}

The demographic data and the presentation reasons for US examination in both groups are detailed in Table I. In Table II the US findings in group 1 are listed. US was reported as normal in 27 patients of group 1, and in 3 of these patients, the abdominal computer tomography (CT) investigation was realized because of continued clinical suspicion. In 2 cases CT was normal while in one case acute appendicitis was diagnosed. The mesenteric lymph nodes detected in US examination were between $33 \times 15$ $\mathrm{mm}$ and $13 \times 7 \mathrm{~mm}$.

Table III. Final diagnosis and treatment methods in patient and control groups

\begin{tabular}{|c|c|c|c|}
\hline \multicolumn{2}{|c|}{ Patient group } & \multicolumn{2}{|l|}{ Control group } \\
\hline Final diagnosis & $\mathrm{n}$ & Final diagnosis & $\mathrm{n}$ \\
\hline Surgery & 56 & Medical treatment & 100 \\
\hline Acute appendicitis & 37 & Normal & 20 \\
\hline Perforated appendicitis & 11 & Disorders of urinary system & 19 \\
\hline Reactive lymphoid hyperplasia & 7 & Upper respiratory tract infection & 14 \\
\hline Invagination & 1 & Irritable bowel syndrome & 11 \\
\hline Medical treatment & 44 & Gastroesophageal reflux & 9 \\
\hline Non-specific diagnosis & 17 & Vitamin deficiency / malabsorption & 7 \\
\hline Gastroenteritis & 17 & Non-specific functional bowel disorder & 7 \\
\hline Mesenteric lymphadenitis & 6 & Endocrine disorders & 5 \\
\hline Invagination & 2 & Dyspepsia & 5 \\
\hline Terminal ileitis & 1 & Lower respiratory tract infection & 3 \\
\hline Intestinal infection & 1 & & \\
\hline Total & 100 & & 100 \\
\hline
\end{tabular}

n, number of patients 
Table III detailed the final diagnoses and the therapeutic approach in both groups.

The presence/absence of ErPC according to the final diagnosesis presented in Table IV and fig 6. The diagnosis of appendicitis, gastroenteritis, mesenteric lymphadenitis, intestinal infection, terminal ileitis and invagination were accepted as causes of intra-abdominal inflammation. The sensitivity of ErPC for intra-abdominal inflammation was calculated to be $80 \%$ and its specificity was $87 \%$.

In Group 2, ErPC was positive only in 3 patients (3\%) with the final diagnoses of lower respiratory tract infection, functional bowel disorder and other disorders of the urinary tract. While WBC level was higher in patients with functional bowel disorder $\left(11.35 \times 10^{9} / \mathrm{L}\right)$, it was within the normal limits in the other 2 patients.

No significant correlation between the presence of ErPC and age $(p=0.103 ; r=0,164)$ sex $(p=0.097 ; r=0.166)$ and CRP $(p=0.063 ; r=0.222)$ could be established. There was a moderate and significant positive correlation between ErPC and WBC $(p=0.010 ; r=0.255)$. In both groups a very good concordance between observers in terms of the presence of ErPC on abdominal US was found (concordance 97\% and kappa 0.93).

\section{Discussions}

ErPC is a rare US finding that manifests itself with the thickening of the periportal area. It is a non-specific US observation and its relation with the underling diseases is relatively unknown. Recognition of this phenomenon, the pathophysiology of which is not proven, is important because it should be considered as a sign of pathological liver. In our study, we found that the presence of ErPC was frequently diagnosed in patients with gastroenteritis, followed by cases diagnosed with

Table IV. Echo-rich periportal cuffing frequency according to final diagnoses in group 1

\begin{tabular}{lll}
\hline Final diagnoses & $\begin{array}{l}\text { ErPC } \\
\text { negative }\end{array}$ & $\begin{array}{l}\text { ErPC } \\
\text { positive }\end{array}$ \\
\hline Non-specific diagnosis & $5(19.2)$ & $12(16.2)$ \\
Mesenteric lymphadenitis & $1(3.8)$ & $5(6.8)$ \\
Acute and perforated appendicitis & $11(42.3)$ & $37(50)$ \\
Gastroenteritis & $1(3.8)$ & $16(21.6)$ \\
Intestinal infection & 0 & $1(1.4)$ \\
Terminal ileitis & 0 & $1(1.4)$ \\
Reactive lymphoid hyperplasia & $6(23.1)$ & $1(1.4)$ \\
Invagination & $2(7.7)$ & $1(1.4)$ \\
Total cases & 26 & 74 \\
\hline
\end{tabular}

The results are expressed as number(\%). ErPC, Echo-rich Periportal Cuffing

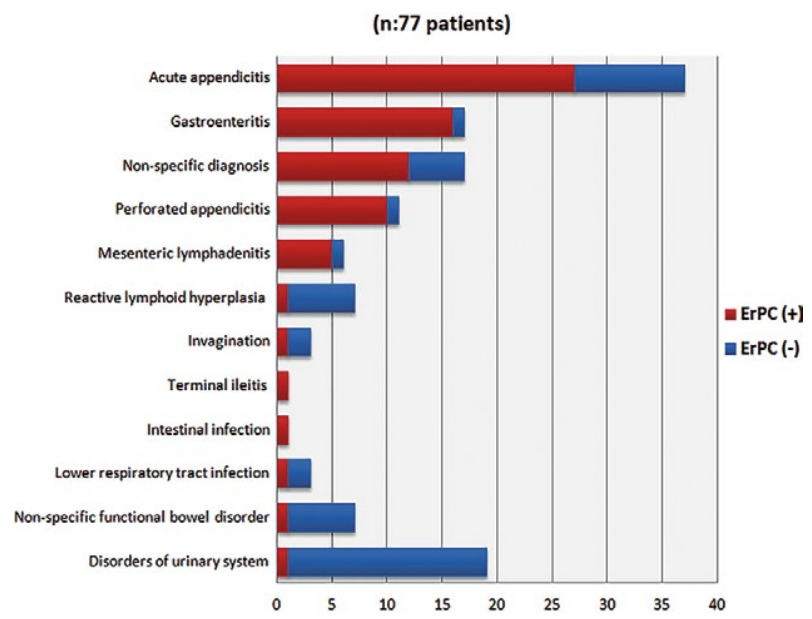

Fig 6. Ranking list of the final diagnoses associated with periportal cuffing in both groups. Horizontal line demonstrates number of the patients. ErPC, echo-rich periportal cuffing; $\mathrm{n}$, number of patients.

perforated appendicitis, mesenteric lymphadenitis and acute appendicitis. ErPC was negative in 6 of 7 patients operated on for suspected acute appendicitis and whose pathology results were reported as reactive lymphoid hyperplasia, as well as in 97 of 100 patients of the control group. According to our results, ErPC it is highly predictable for an abdominal acute inflammatory process. ErPC was positive in each patient diagnosed with invagination, terminal ileitis, and bowel infection but studies with large case series are required since we had very few cases with these pathologies.

Ringler et al retrospectively evaluated US examinations of 1853 patients and Neesse et al retrospectively evaluated US examinations of 10500 patients. The authors reported the presence of hyperechoic PC in 12 $(0.65 \%)$ and 91 patients $(0.86 \%)$, respectively $[4,10]$. We detected ErPC in 3\% of patients in the control group. This higher frequency could be related especially to the pediatric population enrolled in this study compared with adult patients enrolled in the aforementioned study.We found no significant correlation between ErPC and the children's age. The prominent relationship between acute appendicitis, gastroenteritis and mesenteric lymphadenitis and the presence of ErCP, as we found in our pediatric group, was not described in adult patients. Therefore, we think that ErPC is more common in children and may be negatively correlated with age.

Neese et al found that all 14 cases of inflammatory bowel disease displayed ErPC positivity and 12 of them showed significantly higher CRP levels [10]. Similar to the literature, in our study, the mean CRP and WBC levels in the patient group was higher than normal. Particularly, CRP levels were increased in $27 / 37$ patients with 
appendicitis, $9 / 10$ patients with gastroenteritis, all of the 4 patients with mesenteric lymphadenitis, 1 patient with terminal ileitis and in 1 patient with a bowel infection. However, although there was a positive and significant correlation between the presence of ErPC and WBC levels, no correlation was found with CRP levels. This could be related to the low number of cases in some subgroups and the lack of CRP determination in some of our patients. We think that a positive correlation may become apparent in studies carried out with a larger series.

In a retrospective study Tulin-Silver et al compared the US findings of acute appendicitis and perforated appendicitis in pediatric patients [18] and found that the sensitivity and specificity of ErPC was higher in patients with perforated appendicitis $(51.8 \%, 81.3 \%$, respectively). They stated that an increase in periportal echogenicity might reflect the oedematous response of the liver to peritonitis. We found ErPC in $90 \%$ of patients diagnosed with perforated appendicitis and $73 \%$ of patients with acute appendicitis. Interestingly, we also detected a high rate of ErPC positivity in patients diagnosed with gastroenteritis and mesenteric lymphadenitis (94\% and 83\%, respectively). We assume that the reason for the increase in the PC in these inflammatory diseases could be related to the abnormal cells that pass through the portal system by means of enterohepatic circulation from the intestinal mucosa and may cause inflammation in the liver. In perforated appendicitis the ErPC may reflect the edematous response of the liver to peritonitis. Although we had only 1 patient with bowel infection and terminal ileitis, the fact that ErPC was positive in this patient also promotes this hypothesis.

The periportal modification seems to be an US specific finding. In the prospective study of Akata et al [12] on 50 children with cystic fibrosis the increase in periportal echogenicity in 18 patients (37\%) could not be correlated with CT findings.

There are several limitations to our study. A first limitation is that our study groups were not well balanced with respect to gender composition. Since we planned the study prospectively, we included the cases consecutively according to their symptoms and we could not foresee the gender distribution. Due to the fact that we found no significant correlation between ErPC and gender we assumed that this gender difference did not affect our results. The second limitation is that there was the small number of specific cases in some subgroups such as mesenteric lymphadenitis, terminal ileitis and invagination. The lack of follow up of patients with ErPC in order to determine how long the modifications in liver are lasting, the lack of comparison with other imaging techniques and with histology are also limitations of our study.
In conclusion, ErPCappears especially in diseases associated with abdominal inflammation such as gastroenteritis, acute appendicitis, perforated appendicitis and mesenteric lymphadenitis. When evaluated with adequate clinical information, the presence of ErPC is a finding of high sensitivity and specificity in pediatric patients. This could have a significant contribution to correct diagnoses by directing the radiologists or clinicians to further examinations or follow-ups.

\section{References}

1. Abu-Judeh HH. The "starry sky" liver with right-sided heart failure. Am J Roentgenol 2002;178:78.

2. Rak K, Hoper KD, Parker SH. "Starry sky" liver with fasting: variations in glycogen stores? J Ultrasound Med 1996;15:405-407.

3. Siegel MJ. Pediatric sonography. 4th ed., Lippincott Williams \& Wilkins. Philadelphia, PA, 2011.

4. Ringler M, Sturm W, Kathrein H, Judmaier G. Periportal hyperechogenicity of the liver. Clinical aspects and pathology of the so-called fixed star heaven phenomenon of the liver. Ultraschall Med 1997;18:31-34.

5. Neesse A, Heumann T, Görg C, et al. Periportal cuffing in inflammatory bowel diseases: mystery of stars and stripes. Inflamm Bowel Dis 2010;16:1275-1276.

6. Linhart T, Görg C, Moll R, Gress TM. Unusual presentation of autoimmune pancreatitis type 1. Dtsch Med Wochenschr 2010;135:1662-1666.

7. Mortelé KJ, Segatto E, Ros PR. The infected liver: radiologic-pathologic correlation. Radiographics 2004;24:937955.

8. Haliloglu N, Erden A, Erden I. Primary biliary cirrhosis: Evaluation with T2-weighted MR imaging and MR cholangiopancreatography. Eur J Radiol 2009;69:523-527.

9. Karcaaltincaba M, Haliloglu M, Akpinar E, et al. Multidetector $\mathrm{CT}$ and MRI findings in periportal space pathologies. Eur J Radiol 2007;61:3-10.

10. Neesse A, Huth J, Heumann T, et al. Echo-rich and echopoor periportal cuffing: pole position for inflammatory bowel diseases. Ultraschall Med 2008;29:633-638.

11. Molinos Urien AM, Fernandez-Moscoso Lopez-Duran A, Garcia Moreno MA, Herraiz Romero I, Pascual Robles MD, Jaques Perez L. Ultrasound periportal cuffing: differential diagnosis. ECR Congress 2013 Poster No: C-0184. doi: 10.1594/ecr2013/C-0184.

12. Akata D, Akhan O, Ozcelik U, et al. Hepatobiliary manifestations of cystic fibrosis in children: correlation of CT and US findings. Eur J Radiol 2002;41:26-33.

13. Toppet V, Souayah H, Delplace O, et al. Lymph node enlargement as a sign of acute hepatitis A in children. Pediatr Radiol 1990;20:249-252.

14. Park WH, Choi SO, Lee HJ. Technical innovation for noninvasive and early diagnosis of biliary atresia: the ultrasonographic "triangular cord" sign. J Hepatobiliary Pancreat Surg 2001;8:337-341. 
15. Barata CH, Pinto-Silva RA, Lambertucci JR. Abdominal ultrasound in acute schistosomiasis mansoni. Br J Radiol 1999;72:949-952.

16. Chateil J, Brun M, Perel Y, Pillet P, Micheau M, Diard F. Abdominal ultrasound findings in children with hemophagocytic lymphohistiocytosis. Eur Radiol 1999;9:474-477.

17. Schmidt MH, Sung L, Shuckett BM. Hemophagocytic Lymphohistiocytosis in Children: Abdominal US Findings within 1 Week of Presentation. Radiology 2004;230:685689.

18. Tulin-Silver S, Babb J, Pinkney L, et al. The challenging ultrasound diagnosis of perforated appendicitis in children: constellations of sonographic findings improve specificity. Pediatr Radiol 2015;45:820-830.

19. Sanchez TR, Corwin MT, Davoodian A, Stein-Wexler R. Sonography of Abdominal Pain in Children: Appendicitis and Its Common Mimics. J Ultrasound Med 2016;35:627635.
20. Quigley AJ, Stafrace S. Ultrasound assessment of acute appendicitis in paediatric patients: methodology and pictorial overview of findings seen. Insights Imaging 2013;4:741-751.

21. Haber HP, Stern M. Intestinal ultrasonography in children and young adults: bowel wall thickness is age dependent. J Ultrasound Med 2000;19:315-321.

22. Dialer I, Hundt C, Bertele-Harms RM, Harms HK. Sonographic evaluation of bowel wall thickness in patients with cystic fibrosis. J Clin Gastroenterol 2003;37:55-60.

23. Siegel MJ. Pediatric body CT. Wolters Kluwer/ Lippincott Williams \& Wilkins, Philadelphia, PA. 2008.

24. Millet I, Alili C, Pages E, Curros Doyon F, Merigeaud S, Taourel P. Infection of the right iliac fossa. Diagn Interv Imaging 2012;93:441-452.

25. Ruivo C, Portilha MA, Antunes C, et al. Beware of right lower quadrant pain: How ultrasound can help your differential diagnosis. ECR Congress 2010. Poster No: C-1660. doi:10.1594/ecr2010/C-1660 